\title{
Schwieriges Asthma: Klinische Phänotypen und Prinzipien der Therapie
}
G. Menz ${ }^{1}$
R. Buhl²
A. Gillissen ${ }^{3}$
P. $\operatorname{Kardos}^{4}$
H. Matthys ${ }^{5}$
R. Pfister ${ }^{6}$
E. W. Russi ${ }^{7}$
H. U. Simon ${ }^{8}$
C. Vogelmeier ${ }^{9}$
R. Wettengel ${ }^{10}$
H. Worth ${ }^{11}$
K. F. Rabe ${ }^{12}$

\section{Difficult to Manage Asthma: Clinical Phenotypes and Principles of Therapy}

\section{Einleitung}

Die meisten Asthmapatienten können mit den in den aktuellen nationalen und internationalen Therapieempfehlungen diskutierten Kombinationen bewährter Medikamente befriedigend behandelt und ihre Krankheit gut kontrolliert werden.

$2-5 \%$ der Asthmatiker leiden jedoch schätzungsweise an einem sehr schweren, schwierig zu behandelnden Asthma, das auch bei kunstgerechter Anwendung der bekannten Therapieregime inklusive niedriger Dosen oraler Glukokortikoide in der Langzeittherapie nicht ausreichend zu stabilisieren ist [1]. Diese relativ kleine Gruppe von Patienten verursacht einen unverhältnismäßig großen Anteil direkter und indirekter Gesundheitskosten [2]. Die Lebensqualität dieser Patienten ist erheblich beeinträchtigt. Sie beanspruchen die ambulanten und stationären Einrichtungen des Gesundheitssystems häufig und intensiv und leiden vielfach unter erheblichen Folgeschäden der Therapie.

In letzter Zeit haben sich in vielen Ländern Expertengremien in Workshops mit schwierigem Asthma beschäftigt. Zudem sind diverse multizentrische Projekte angelaufen.

In Europa wurde die ERS (European Respiratory Society) „Task Force on Difficult/Therapy - Resistent Asthma" gegründet [3]. In den USA wurde ein Workshop des National Heart, Lung and Blood Institute (NHLBI) über „Pathophysiology of Severe Asth- ma" durchgeführt [4] ENFUMOSA (European Network For Understanding Mechanism Of Severe Asthma) ist ein multizentrisches Projekt, an dem 13 Zentren in 9 europäischen Ländern teilnehmen, PARAPLU ein Projekt der Universität Leiden (NL) in Kooperation mit pneumologischen Polikliniken in Süd-Holland, bei dem eine Gruppe von schwierigen Asthmatikern genau studiert und prospektiv verfolgt wird. Erste Ergebnisse einer Querschnittsuntersuchung dieser Population sind inzwischen publiziert [5-7].

Im Rahmen der „3. Davoser Gespräche“ diskutierten die Autoren der vorliegenden Arbeit die vorhandene Literatur und erarbeiteten Vorschläge zum Management des schwierigen Asthma.

\section{Definition}

Eine Vielzahl von verschiedenen Begriffen wird in der Praxis verwendet, um Patienten mit schwierigem Asthma zu charakterisieren: „Akut schwieriges Asthma, chronisch schwieriges Asthma, akut schweres Asthma, chronisch schweres Asthma, therapieresistentes Asthma, therapierefraktäres Asthma, schwer behandelbares Asthma, kortikosteroidpflichtiges Asthma, kortikosteroidresistentes Asthma, lebensbedrohliches Asthma, fatales Asthma, Brittle Asthma“ etc. [1,3,8,9].

${ }^{1}$ Hochgebirgsklinik Davos-Wolfgang, $\mathrm{CH}$

${ }^{2}$ Pneumologie Johannes Gutenberg-Universität, Mainz

${ }^{3}$ Robert-Koch-Klinik, Leipzig

${ }^{4}$ Pneumologie/Allergologie Praxis, Frankfurt/M.

${ }^{5}$ Pneumologie Universitätsklinikum Freiburg

${ }^{6}$ Pneumologie/Allergologie Praxis, Überlingen

${ }^{7}$ Pneumologie Universitätsspital, Zürich

${ }^{8}$ Pharmakologisches Institut Universität, Bern

${ }^{9}$ Pneumologie Philipps-Universität, Marburg

${ }^{10}$ Karl-Hansen-Klinik, Bad Lippspringe

${ }^{11}$ Med. Klinik, Fürth

${ }^{12}$ Dep. Pulmology, Leiden, NL

Korrespondenzadresse

PD Dr. med. G. Menz · Hochgebirgsklinik Davos-Wolfgang · 7265 Davos-Wolfgang · Schweiz

E-mail: guenter.menz@hgk.ch 
Wir haben für diese Übersicht den Ausdruck „schwieriges“ Asthma gewählt, da er unseres Erachtens prägnant die angesprochene Problematik am besten wiedergibt. Es handelt sich hierbei um alle Formen eines schweren oder auch schwer zu behandelnden Asthma. Im Folgenden werden wir auf die internationalen Bemühungen zu einer verständlichen Definition eingehen.

In der Tab. 1 sind die Faktoren angeführt, die der NHLBI-Workshop für die Bewertung des Schweregrades und der Definition von „Severe Asthma“ als wichtig betrachtet [9].

Tab. 1 Faktoren für die Bewertung des Schweregrades eines Asthma

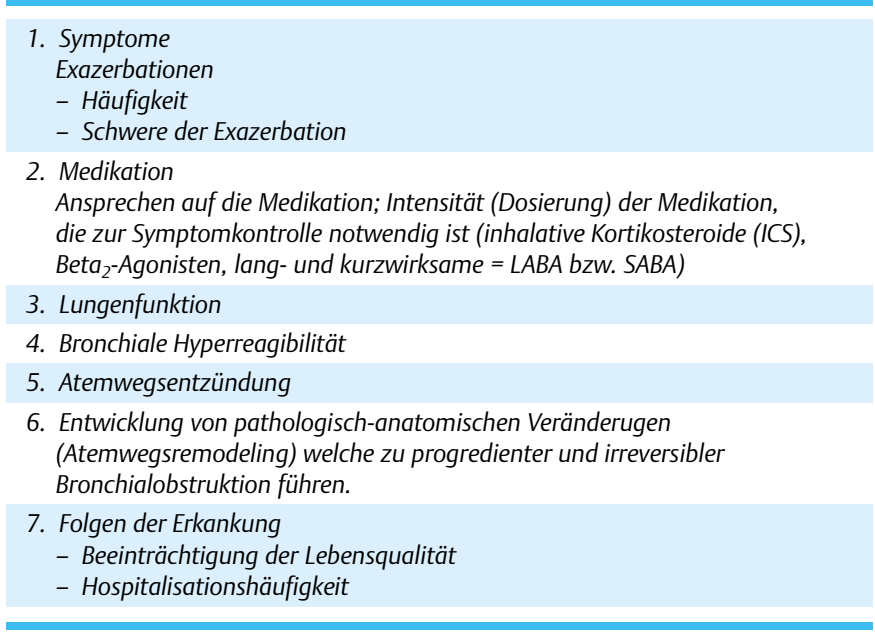

\section{Faktoren für die Bewertung des Schweregrades eines Asthma}

Bei der ENFUMOSA-Definition des schwierigen Asthma, werden die Patienten in 3 Gruppen eingeteilt. Gruppe 1 umfasst Patienten, die trotz hoher Dosen an inhalativen Glukokortikosteroiden und langwirksamen inhalativen Beta ${ }_{2}$-Agonisten symptomatisch sind und häufige Exazerbationen erleben. Die 2. Gruppe bildeten Patienten mit einer ständig notwendigen systemischen Steroidtherapie. Die 3. Gruppe setzt sich aus Patienten mit lebensbedrohlichen Asthmaanfällen (evtl. mit Intubation) in der Anamnese zusammen. Die für die ENFUMOSA-Studie rekrutieren Patienten sollten mindestens drei von fünf der in Tab. 2 angeführten Kriterien erfüllen.

Tab. 2 Definition: Schwieriges Asthma (ENFUMOSA)

Patienten sollten mindestens drei der folgenden Kriterien erfüllen:

- Betreuung durch Spezialisten in den letzten zwei oder mehr Jahren

- persistierende Symptome und deutlich eingeschränkte Lebensqualität

- maximale Asthmatherapie nach gültigen Richtlinien, inkl. hochdosierte inhalative Kortikosteroide und gesicherte Therapie-Compliance

- Episoden von schwerer respiratorischer Insuffizienz/Intubation/Reanimation

- wiederholte Lungenfunktionseinschränkung mit FEV $1<70 \%$ vom Sollwert

\section{Definition: Schwieriges Asthma}

Die zuverlässige Einstufung eines Asthmapatienten als Patient mit schwierigem Asthma kann nicht gestützt auf eine einzige Untersuchung erfolgen, selbst nicht durch einen Spezialisten. Eine Beobachtungs- und Behandlungszeit von 6-12 Monaten ist dazu in der Regel nötig. Die Beurteilung, ob tatsächlich ein schwieriges Asthma vorliegt, sollte immer durch einen in der Behandlung von Asthma erfahrenen Arzt (Pneumologen) erfolgen $[3,8,9]$. In der Beobachtungszeit sollte sich die Therapie auf die allgemein bekannten Empfehlungen zur Asthma Behandlung stützen. Zudem muss die Compliance des Patienten als einwandfrei dokumentiert werden.

Die Diagnose schwieriges Asthma wird auf der Basis folgender Gesichtspunkte gestellt:

Schwieriges Asthma ist definiert durch anhaltende Beschwerden, persistierende Atemwegsobstruktion bzw. hochgradige Variabilität der Atemwegsobstruktion und einen ständigen und häufigen Gebrauch von Beta ${ }_{2}$-Agonisten (Reliever), trotz regelmäßiger Anwendung einer ausgeschöpften Langzeittherapie mit inhalativen Steroiden und langwirksamen inhalativen Be$\mathrm{ta}_{2}$-Agonisten sowie dem zusätzlichen Einsatz von Reservemedikamenten (z. B. Theophyllinen, Leukotrienantagonisten). Die Patienten benötigen immer wieder oder sogar dauernd systemisch verabreichte Steroide. Eine völlige oder weitgehende Wirkungslosigkeit der Kortikosteroide (Steroidresistenz) ist selten [10,11].

Die notwendige Diagnostik und Differenzialdiagnostik des schwierigen Asthma wird weiter unten besprochen.

\section{Phänotypen des schwierigen Asthmas}

Unterschiedliche Krankheitsverläufe können als schwieriges Asthma imponieren.

Manche Patienten leiden unter einem schweren Verlauf der Erkrankung über ihr ganzes Leben, manche entwickeln eine plötzliche Progredienz, ohne dass die Ursachen offensichtlich sind.

- Eine allergische Komponente ist bei einem Großteil der Patienten anamnestisch offenkundig, viele von diesen entwickeln aber aus dem allergischen Asthma heraus eine zunehmende, sog. „Intrinsic Asthma-Komponente“, die für die Schwere des Verlaufes der Erkrankung immer bedeutsamer wird.

- Nichtallergisches Asthma: Manche Patienten waren bis in das mittlere Erwachsenenalter gesund und erkrankten dann plötzlich schwer. Viele von diesen Patienten geben an, die Krankheit habe sich nach einem Infekt der Atemwege entwickelt oder sei erstmals nach Einnahme von nicht steroidalen antiinflammatorischen Substanzen (NSAID), meist Aspirin aufgetreten. Bei diesen Patienten ist eine Allergie meistens nicht nachweisbar. Sie entwickeln das typische intrinsische Asthma, das relativ häufiger einen schweren steroidpflichtigen Verlauf nimmt.

- Patienten mit schwierigem Asthma und Produktion von eosinophilem Sputum stehen Patienten ohne oder mit wenig Sputumproduktion und teils mit neutrophiler Entzündung gegenüber $[1,9]$. 
- Eine Subgruppe der Patienten hat häufig nicht vorhersehbare Episoden von extremer, lebensbedrohlicher, sehr plötzlicher Atemwegsobstruktion bei ansonsten normal verlaufenden Asthma (Brittle Asthma) [13].

Die Tab. 3 fasst Kennzeichen des schwierigen Asthmas zusammen, wie sie sich aus einer Querschnittsuntersuchung der ENFUMOSA-Studie ergaben.

Tab. 3 Kennzeichen des schwierigen Asthma (ENFUMOSA)

- Prädominanz des weiblichen Geschlechtes

- häufig nicht-allergische Asthmaformen (Intrinsic Asthma)

- Analgetikaintoleranz als Risikofaktor

- Assoziation mit Übergewicht (besonders bei Frauen)

- fixierte Atemwegsobstruktion, dauernd erhöhter RV- und/oder TLC-Wert (Hinweis auf strukturelle Veränderungen)

Die Beteiligung der oberen Atemwege im Sinne der rezidivierenden hyperplastischen Rhinosinopathie (extensive sinus disease) hat eine große Bedeutung bei schwierigem Asthma [14].

Gibt es Faktoren, die zur Entwicklung des schwierigen Asthma disponieren?

Genetische Studien zum Asthma sind in den letzten Jahre vorangetrieben worden. „Kandidaten-Gene“ sind in Zusammenhang mit Asthma gebracht worden. Bis heute gibt es aber keine sicheren Erkenntnisse über den Einfluss von bestimmten Genen auf den Phänotyp des Asthma und die Schwere des Krankheitsverlaufes [15].

\section{Allergene}

Die Bedeutung verschiedener Allergene und die Intensität der Allergenexposition für die Entwicklung von Asthma ist belegt [16-18]. Die allergische Sensibilisierung gegenüber Alternaria alternata wird als Risikofaktor für schwieriges Asthma angesehen [18]. In der Untersuchung von Grootendorst sind jedoch bei schwierigem Asthma Typ I-Sensibilisierungen gegen Hausstaubmilben, Tierhaare, Gräserpollen und Aspergillus fumigatus deutlich häufiger als gegen Alternaria beobachtet worden (PARAPLU) [19].

Im Gegensatz dazu zeigt ENFUMOSA, dass Allergene bei Patienten mit schwierigem Asthma eher von untergeordneter Bedeutung sind.

\section{Respiratorische Infekte}

Bei Kindern sind Infektionen mit Respiratory Syncitial Virus (RSV) und Parainfluenza-Infektionen in Zusammenhang mit der Entwicklung eines Asthma gebracht worden [20,21]. Bei Kindern und Erwachsenen lösen virale Infekte Exazerbationen aus [22-25]. Mykoplasmeninfektionen und Adenovireninfektionen sollen sowohl für Exazerbationen als auch für die längerfristige Progredienz eines Asthmaverlaufes verantwortlich sein $[26,27]$. Diskutiert wird die Rolle von Chlamydieninfektionen sowohl für die Verschlechterung des Asthmaverlaufes [28] als auch für die persistierende Atemwegsobstruktion des schweren IntrinsicAsthmatikers [29].

\section{Glukokortikoidresistenz}

Glukokortikosteroide sind bis heute die wirksamsten Medikamente zur Behandlung des Asthma. Einige wenige Patienten reagieren jedoch nicht adäquat auf die üblich angewandten Dosierungen (Relative Glukokortikoidresistenz). Man unterscheidet verschiedene Formen von Glukokortikoidresistenz [30-32], auf die an dieser Stelle nicht näher eingegangen werden soll.

Neben dem Ausbleiben einer günstigen Wirkung auf den klinischen Verlauf und die Lungenfunktion sind fehlende Kortikoideffekte im peripheren Blutbild und in der Analyse von Lymphozytensubpopulationen und Lymphozyten- und Monozytenaktivierungsmarkern Zeichen der (relativen) Kortikoidresistenz [33]. Interferon- $\alpha$ (INF- $\alpha$ ) ist bei Patienten mit Steroidresistenz möglicherweise in der Lage diese zu modulieren und damit zu durchbrechen [34].

\section{Sicherung der Diagnose und Ausschluss anderer Erkrankungen}

Bevor die Arbeitsdiagnose des schwierigen teils therapierefraktären Asthma gestellt werden kann, muss die Diagnose Asthma eindeutig gesichert sein. Zum Ausschluss anderer Krankheiten bzw. zur Erfassung zusätzlicher Erkrankungen ist eine gründliche systematische Abklärung notwendig. Diese Untersuchungen sollen durch Spezialisten durchgeführt werden. Sie erfordern allenfalls einen stationären Aufenthalt des Patienten an einem auf Asthma spezialisierten Zentrum.

Zur Charakterisierung des Patienten mit schwierigem Asthma ist ein regelrechtes Staging der Erkrankung notwendig. Nicht alle der in den Abb. 1 und $\mathbf{2}$ angegebenen Untersuchungen sind bei jedem Patienten sinnvoll und notwendig! Die Arbeitsdiagnose schwieriges Asthma wird unseres Erachtens am besten wie in Abb.1 angegangen. Ist die Diagnose schwieriges Asthma bestätigt, sollte nach Abb. 2 vorgegangen werden.

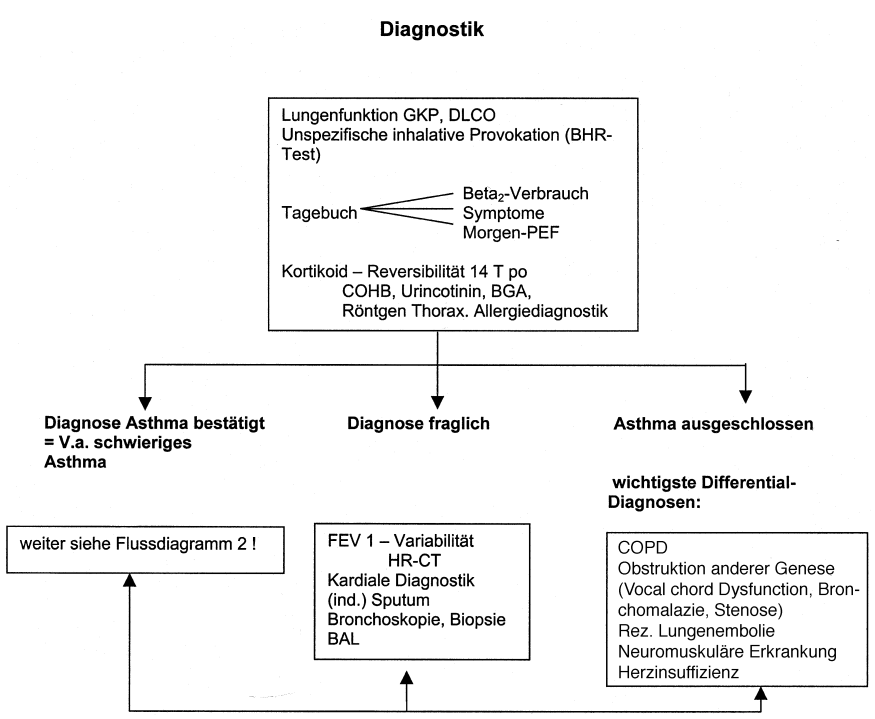

Abb. 1 Arbeitsdiagnose Schwieriges Asthma. 


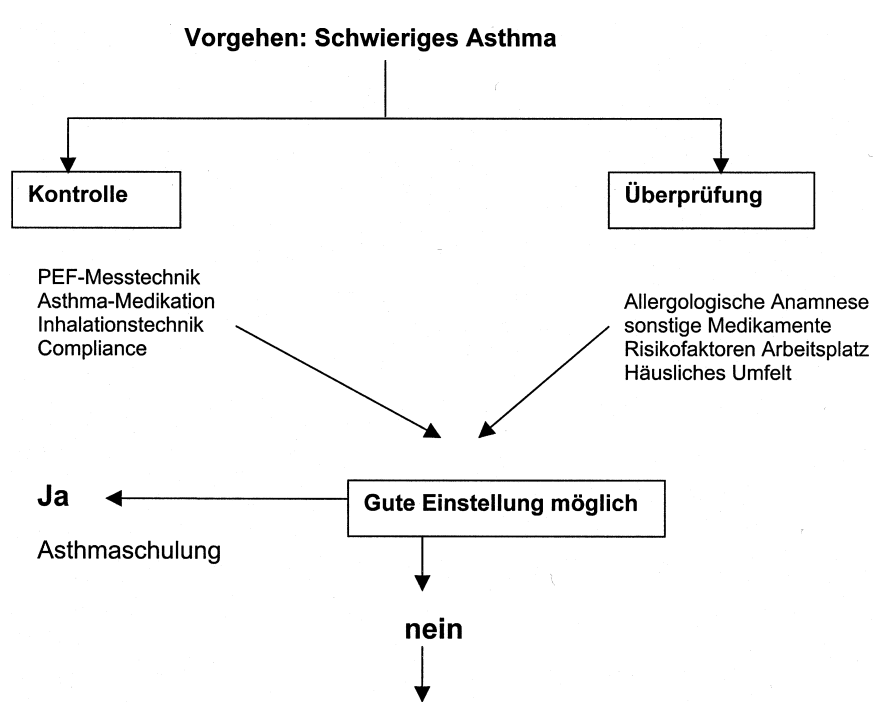

Weiterführende Diagnostik:

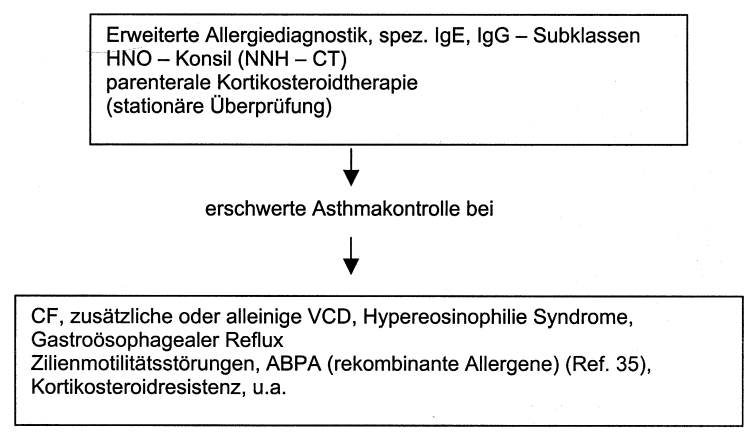

Abb. 2 Vorgehen schwieriges Asthma.

Legende zu den Abkürzungen: $A B P A=$ Allergische bronchopulmonale Aspergillose, $\mathrm{BAL}=$ Bronchoalveoläre Lavage, $\mathrm{BGA}=$ Blutgasanalyse , $\mathrm{BHR}=$ Bronchiale Hyperreagibilität, $\mathrm{CF}=$ Cystische Fibrose, $\mathrm{DLCO}=$ Diffusionskapazität, GKP = Ganzkörperplethysmographie, HRCT = HighResolution-Computer-Tomographie, $\mathrm{PEF}=$ Peak-Flow, $\mathrm{VCD}=$ Vocal Cord Dysfunktion.

Patientenmanagement und Therapieansätze bei schwierigem Asthma

Neben den medikamentösen Möglichkeiten sind die Beeinflussung psychosozialer Faktoren, die Patientenschulung, Ansätze zur Verbesserung der Compliance und der Inhalationstechniken, eine möglichst weitgehende Allergenkarenz und Meidung von Substanzen, auf die der Patient ungünstig reagierte, von hoher Bedeutung. Darüber hinaus sollte bei diesen Patienten auf eine strikte Nikotinkarenz geachtet werden (aktiv und passiv!). Basis der medikamentösen Therapie bleiben auch bei schwierigem Asthma die hochdosierten inhalativen Glukokortikosteroide der jüngsten Generation in Kombination mit langwirksamen inhalativen Beta ${ }_{2}$-Sympathikomimetika (Formoterol, Salmeterol). Wie Grootendorst u. Mitarb. zeigten, führt eine kurze Periode der weitgehenden Allergenkarenz im Hochgebirge zusätzlich zu o.g. optimierter Medikation zu einer markanten Verbesserung gemessen an klinischen Parametern und Inflammationsmarkern. Daraus resultiert die Verbesserung der Langzeitkontrolle des schwierigen Asthmas über das medikamentös Erreichte hinaus und zusätzlich eine Reduktion der systemischen Steroiddosis [36].
In den letzten Jahren wurden immer wieder Versuche mit verschiedenen Medikamenten, meistens Immunsuppressiva gemacht, um die oft sehr hohen oralen Steroiddosen bei Patienten mit schwierigem Asthma zu reduzieren. Die Studien mit Methotrexat, Cyclosporin A, Colchicin, Troleandomycin (TAO), i. v. Lidocain, Heparin, Dapsone und Goldsalzen zeigen unterschiedliche, im längeren Verlauf meist enttäuschende Ergebnisse [37]. Allerdings beobachtete man immer wieder sogenannte,,Responder“, besonders mit Cyclosporin A aber auch mit Methotrexat. Erste Ergebnisse der Immunmodulation mit Interferon-Alpha (INF- $\alpha$ ) zeigten positive Resultate [34]. Weitere kontrollierte Untersuchungen sind jedoch unbedingt erforderlich, bevor diese Therapieform breiter eingesetzt werden darf. Eine hohe Dosierung von Zafirlukast ( 80 mg BID) ergab eine bessere Kontrolle von Patienten, die unter einer hohen Dosis von inhalativen Steroiden $(1000-4000 \mu \mathrm{g} / \mathrm{Tag})$ instabil waren [38].

Therapieansätze mit Anti-Interleukin 5, Anti-Interleukin 4-Rezeptor, Anti-Adhäsionsmolekülen etc. sind derzeit nicht ausreichend untersucht und die vorläufigen Ergebnisse sind eher enttäuschend [39]. Einzig die Behandlung mit einem monoklonalen Antikörper gegen IgE (E-25, Omalizumab) zeigte in ersten klinischen Untersuchungen bei Patienten mit einer allergischen Rhinitis und allergischem Asthma einen gewissen Effekt [40,41]. Es fehlen jedoch auch bei dieser noch nicht zugelassenen Therapieform Langzeitstudien, insbesondere bei Patienten mit einer schwereren Verlaufsform der Erkrankung.

Abschlussbemerkung: Es kann nicht oft genug betont werden, dass gerade bei den in dieser Arbeit angesprochenen Patienten sehr intensiv und immer wieder nach fehlerhafter Diagnose, mangelnder Compliance, Anwendungsfehlern der inhalativen Medikation und nicht erkannter bzw. negierter Allergenexposition und Infektgefährdung (z. B. im beruflichen Umfeld) gefahndet werden muss (siehe Tab. 4-5)!

\section{Gedanken zu zukünftigen Therapiestrategien}

Die verschiedenen Phänotypen des schwierigen Asthmas erfordern unterschiedliche Therapiestrategien.

Ziele der strategischen Überlegungen zur Therapie müssen sein:

1. Prävention der plötzlichen, lebensgefährlichen Attacken bei „Brittle Asthma.“

2. Prävention bzw. Stopp des progredienten und irreversiblen Lungenfunktionsverlustes, Beeinflussung von Atemwegsremodeling.

3. Erforschung der Mechanismen, die zu wiederholten Exazerbationen führen.

4. Inhibition der trotz der systemischen Kortikosteroidtherapie persistierenden eosinophilen und/oder neutrophilen Infiltration.

5. Wiederherstellung der Steroidempfindlichkeit bei Steroidresistenz und darüber hinaus Erforschung bisher unbekannter Entzündungsprozesse und -mechanismen. 


\section{Therapieoptionen}

\section{$\begin{array}{lll}\text { A) Optimierung der „Basismedikation“ } & \text { B) Ausschalten von ungünstigen Triggern } & \text { C) Diagnostik und Ausschalten von Faktoren, }\end{array}$} die Exazerbationen begünstigen:

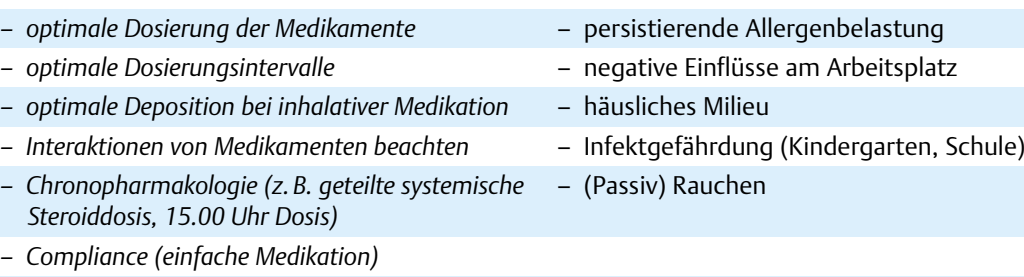

- persistierende Allergenbelastung

- negative Einflüsse am Arbeitsplatz

- häusliches Milieu

- Infektgefährdung (Kindergarten, Schule)

- (Passiv) Rauchen

\author{
- seltene Allergene \\ - gastroösophagealer Reflux (24 h-pH-Metrie, Gastroskopie) \\ - rez. NNH-Affektionen (CT-NNH, Endoskopie) \\ - Infektionen des Respirationstraktes \\ - Begleiterkrankungen (Hyperthyreose, Churg Strauss- \\ Syndrom, ABPA, VCD, etc.) \\ - Menstruationsphasen \\ - Medikamente (Betablocker, NSAID, ACE-Hemmer [Husten])
}

Tab. 5 Schwieriges Asthma

\section{Glukokortikosteroidresistenz}

\section{- „absolute“ Resistenz: selten!}

- parenterale Therapie zur Überprüfung erforderlich

- Blutbild, eosinophile Leukozyten, Immunstatus

- relative Steroidresistenz

- Therapie (siehe Text)

\section{Literatur}

${ }^{1}$ Barnes PJ, Woolcock AJ. Difficult asthma. Eur Respir J 1998; 12: $1209-1218$

2 Barnes PJ, Jonsson B, Klim JB. The costs of asthma. Eur Respir J 1996; 9: 636-642

${ }^{3}$ Chung KF et al. For the ERS Task Force on Difficult/Therapy - Resistant Asthma Difficult/therapy - resistant Asthma. Eur Respir J 1999; 13: $1198-1208$

${ }^{4}$ Busse WW, Banks-Schlegel S, Wenzel SE. Pathophysiology of severe asthma. J Allergy Clin Immunol 2000; 106: 1033 - 1042

5 ten Brinke A, van Dissel JT, Sterk PJ, Zwinderman AH, Rabe KF, Bel EH. Persistent Airflow Limitation in Adult-onset Nonatopic Asthma is associated with Serological Evidence of Chlamydia Pneumonieae Infection. J Allergy Clin Immuol 2001; 107: 449-454

${ }^{6}$ ten Brinke A, Zwinderman AH, Sterk PJ, Rabe KF, Bel EH. Factors associated with Airflow Limitation in Severe Asthma. Am J Respir Crit Care Med 2001; 164: 744-748

7 ten Brinke A, de Lange C, Zwinderman AH, Rabe KF, Sterk PJ, Bel EH. Sputum Induction in Severe Asthma by a Standardized Protocol. Predictors of Excessive Bronchoconstriction. Am J Respir Crit Care Med 2001; 164: 749-753

${ }^{8}$ Anonymous. Guidelines for the diagnosis and management of asthma. Expert panel report 2. Bethesda (MD); NIH 1997; Publication No.: 97-4051

${ }^{9}$ Wenzel SE, Fahy JV, Irvin C, Peters SP, Spector S, Szefler S. Proceedings of the ATS Workshop on Refractory Asthma: current understanding, recommendations and unanswered questions. Am J Respir Crit Care Med 2000; 162: $2341-2351$

10 Woolcock AJ. Steroid resistant asthma: What is the clinical definition? Eur J Respir Dis 1993; 6: $743-747$

11 Thomas PS, Geddes DM, Barnes PJ. Pseudo-steroid resistant asthma. Thorax 1999; 54: $352-356$

12 Wenzel SE, Schwartz LB, Langmack EL, Halliday JL, Trudeau JB, Gibbs $R L$ et al. Evidence that severe asthma can be divided pathologically in two inflammatory subtypes with distinct physiologic and clinical characteristics. Am J Respir Crit Care Med 1999; 160: 1001 - 1008

${ }^{13}$ Ayres JG, Miles JF, Barnes PJ. Brittle asthma. Thorax 1998; 53: 315-321
${ }^{14}$ Bresciani M, Paradis L, Des Roches A, Vernhet H, Vachier I, Godard P, Bousquet J, Chanez P. Rhinosinusitis in severe asthma. J Allergy Clin Immunol 2001; 107 (1): $73-80$

${ }^{15}$ Wiesch DG, Meyers DA, Bleecker ER. Genetics of asthma. J Allergy Clin Immunol 1999; 104: 895-901

${ }^{16}$ Sporik R, Holgate ST, Platts-Mills TAE, Cogswell JJ. Exposure to housedust mite allergene (Der p.I.) and the development of asthma in childhood. N Engl J Med 1990; 323: 502-507

${ }^{17}$ Squillace SP, Sporik RB, Rakes G, Couture N, Lawrence A, Merriam S et al. Sensitization to dust mites as a dominant risk factor for asthma among adolescents living in central Virginia. Multiple regression analysis of a population - based study. Am J Respir Crit Care Med 1997; 156: $1760-1764$

${ }^{18}$ Neukirch C, Henry C, Leynaert B, Liard R, Bousquet J, Neukirch F. Is sensitization to Alternaria alternata a risk factor for severe asthma? A population-based study. J Allergy Clin Immunol 1999; 103: 709-711

19 Grootendorst DC, ten Brinke A, Sterk PJ, Rabe KF, Bel EH. Different allergic profile in patients with early and late onset severe asthma. Am J Respir Crit Care Med 2000; 160: A323

${ }^{20}$ Martinez FD, Wright AL, Taussig LM, Holberg CJ, Halonen M, Morgan WJ et al. Asthma and wheezing in the first six years of life. $\mathrm{N}$ Engl J Med 1995; 332: 133-138

${ }^{21}$ Stein RT, Sherrill D, Morgan WJ, Holberg CJ, Halonen M, Taussig LM et al. Respiratory syncytial virus in early life and risk of wheeze and allergy by age 13 years. Lancet 1999; 354: $541-545$

${ }^{22}$ Folkerts G, Busse WW, Nijkamp FP, Sorkness R, Gern JE. Virus - induced airway hyperresponsiveness and asthma. Am J Respir Crit Care Med 1998; 157: $1708-1720$

${ }^{23}$ Johnston SL, Pattemore PK, Sanderson G, Smith S, Lampe F, Josephs LK et al. Community study of role of viral infections in exacerbations of asthma in 9-11-year old children. BMJ 1995; 310: 1225-1228

${ }^{24}$ Johnston SL, Pattemore PK, Sanderson G, Smith S, Campbell MJ, Josephs $\mathrm{LK}$ et al. The relationship between upper respiratory infections and hospital admissions for asthma: a time-trend analysis. Am J Respir Crit Care Med 1996; 154: 654-660

${ }^{25} \mathrm{Hogg}$ JC. Childhood viral infection and the pathogenesis of asthma and chronic obstructive lung disease. Am J Respir Crit Care Med 1999; 160: S26-28

${ }^{26}$ Kraft M, Cassell GH, Henson JE, Watson H, Wiliamson J, Marmion BP et al. Detection of Mycoplasma pneumoniae in the airways of adults with chronic asthma. Am J Respir Crit Care Med 1998; 158: 998 - 1001

${ }^{27}$ Matsuse T, Hayashi S, Kuwano K, Keunecke H, Jefferies WA, Hogg JC. Latent adenoviral infection in the pathogenesis of chronic airways obstruction. Am Rev Respir Dis 1992; 146: 177-184

${ }^{28}$ Hahn DL. Chlamydia pneumoniae, asthma, and COPD: what is the evidence? Ann Allergy Asthma Immunol 1999; 83: 271 - 292

29 ten Brinke A, van Dissel JT, Sterk PJ, Zwinderman AH, Rabe KF, Bel EH. Persistent airflow limitation in adult - onset nonatopic asthma in associated with serologic evidence of Chlamydia pneumoniae infection. J Allergy Clin Immunol 2001; 107: 449-454

${ }^{30}$ Sher ER, Leung DYM, Surs W, Kam JC, Zieg G, Kamada AK et al. Steroid - resistant asthma - cellular mechanisms contributing to inadequate response to Glucocorticoid therapy. J Clin Invest 1994; 93: 33 - 39 
${ }^{31}$ Leung DYM, Hamid Q, Vottero A, Szefler SJ, Surs W, Minshall E et al. Association of glucocorticoid insensitivity with increased expression of glucocorticoid receptor beta. J Exp Med 1997; 186: 1567 - 1574

32 Sousa AR, Lane SJ, Cidlowski JA, Staynov DZ, Lee TH. Glucocorticoid resistence in Asthma is associated with elevated in vivo expression of the Glucocorticoid receptor beta - isoform. J Allergy Clin Immunol 2000; 105: $943-950$

33 Oehling AG, Akdis CA, Schapowal A, Blaser K, Schmitz M, Simon H-U. Suppression of the immune system by oral glococorticoid therapy in bronchial asthma. Allergy 1997; 52: $144-154$

${ }^{34}$ Gratzl S, Palca A, Schmitz M, Simon HU. Treatment with IFN-alpha in corticosteroid-unresponsive asthma. J Allergy Clin Immunol 2000; 105: 1035 - 1036

35 Menz G, Willer G, Crameri R. Die allergische bronchopulmonale Aspergillose (ABPA). Pneumologie 2000; 54: 375-384

${ }^{36}$ Grootendorst DC, Dahlén S-E, van den Bos JW, Duiverman EJ, VeselicCharvat M, Vrijlandt EJLE, O’Sullivan S, Kumlin M, Sterk PJ, Roldaan AC. Benefits of high altitude allergen avoidance in atopic adolescents with moderate to severe asthma, over an above treatment with high dose inhaled steroids. Clinical and Experimental Allergy 2000; 31: 400-408

${ }^{37}$ Gillissen A, Schäfer H, Tasci S, Ewig S. Alternativen zur Standardtherapie des Asthma bronchiale. Dtsch Med Wschr 2000; 125: 1575 - 1579

38 Virchow Jr C, Prasse A, Naya I, Summerton L, Harris A and the Zafirlukast Study Group. Zafirlukast Improves Asthma Control in Patients receiving High-Dose Inhaled Corticosteroids. Am J Respir Crit Care Med 2000; 162: $578-585$

${ }^{39}$ Schmidt DT, Rabe KF. Reliable and modern therapy of allergic bronchial asthma. Der Internist 2000; 41 (12): 1391 - 1398

40 Soler M, Matz J, Townley R, Buhl R, O’Brien J, Fox H, Thirlwell J, Related Articles Gupta N, Della Cioppa G. The anti-IgE antibody omalizumab reduced exacerbations and steroid requierement in allergic asthmatic. Eur Respir J 2001; 18 (2): 254-261

41 Busse W, Corren J, Lanier BQ, McAlary M, Fowler-Taylor A, Cioppa GD, von As A, Gupta N. Omalizumab, anti-IgE recombinant humanized monoclonal antibody, for the treatment of severe allergic asthma. J Allergy Clin Immunol 2001; 108 (2): 184-190

\section{Buchbesprechung}

\section{Tabakabhängigkeit \\ Gesundheitliche Schäden durch das Rauchen \\ Ursachen - Folgen - Behandlungsmöglichkeiten - Konsequenzen für Politik und Gesellschaft \\ Knut-Olaf Haustein}

Deutscher Ärzte-Verlag, Köln 2001. Preis EUR 39,88

ISBN 3-7691-0390-4

Knut-Olaf Haustein, Pharmakologe, Gründer der Deutschen Gesellschaft für Nikotinforschung und Leiter des Instituts für Nikotinforschung und Raucherentwöhnung, legt ein 478seitiges Kompendium zum Thema Inhalationsrauchen und seiner Folgen vor. Sorgfältig recherchiert, prägnant formuliert, graphisch und didaktisch (77 Abbildungen und 53 Tabellen) gut aufbereitet präsentiert sich „Tabakabhängigkeit“ als der deutschsprachige Leitfaden zu Ursachen, Folgen, Behandlungsmöglichkeiten und Konsequenzen für Politik und Gesellschaft.

Die „Geschichte des Tabaks“ ist ein amüsantes Essay zum Vordringen des Rauchgebarens in der alten und modernen Welt. Epidemiologische Zusammenhänge werden auf der Grundlage akutellen Zahlenmaterials erläutert. Tabakinhaltsstoffe werden eingehend besprochen - die Inhaltsstoffe des frischen, unverdünnten Hauptstromrauches würden als noxae non gratae an sich schon genügen, bei Lektüre der Zusammenstellung einiger Tabakinhaltsstoffe für die Zigarettenherstellung und ihre Wirkungen auf den Raucher verschlägt es einem die Sprache.

Haustein äußert sich ausführlich zu Pharmakologie und Pharmakokinetik des Nikotins, erläutert Zusammenhänge zwischen Rauchen und Lungenerkrankungen sowie Herz-Kreislauferkrankungen und Störungen von Blutgerinnungs- und Fibrinolysevorgängen. Andere Organsysteme, wie ZNS, Augen, Ohren, Cavum oris und Larynx, Stoffwechsel, Gastrointestinaltrakt, Urogenitaltrakt, Stützgewebe, Haut und blutbildende Organe werden nicht ausgespart.

Besondere Sorgfalt ist dem Kapitel „Rauchen und Schwangerschaft" gewidmet. Effekte des Rauchens auf die Plazenta, Schwangerschaftskomplikationen, das Problem möglicher Fehlbildungen, Rauchen und plötzlicher Kindstod (SIDS), die Epidemiologie dazu ist bedrückend, fetotoxische Wirkungen von Kohlenmonoxid, Rauchen und Stillperiode, Passivrauchen und Still- periode, Passivrauchen während und nach der Schwangerschaft und Wirkungen von Nikotin werden eingehend und verständlich abgehandelt. Aus pneumologisch-allergologischer Sicht wäre eine breitere Darstellung der immunologisch-allergologischen Konsequenzen des Rauchens wünschenswert gewesen. Allerdings wird dies im Kapitel „Passivrauchen“ durch eine Fülle epidemiologischer Daten nahezu wettgemacht.

Haustein setzt sich mit den nichtmedikamentösen Verfahren zur Raucherentwöhnung und der medikamentösen Behandlung des Rauchers akribisch auseinander und vergisst dabei die Belange der Primärprävention nicht. Letztere zielen auf eine wesentliche stärkere Verantwortung des Einzelnen, der Wirtschaft, der Massenmedien und des Staates bei der Primärprävention, v.a. bei Adoleszenten, ab. Erprobte Modelle der Primärprävention aus den USA und Deutschland, so das Gesundheitsförderungsprogramm Klasse 2000 und das Gesundheitsprogramm der Universität Trier, werden ausführlich beschrieben.

Zu Tabakindustrie und Werbung, Gesellschaft, Politik und Tabakindustrie legt Haustein interessante Fakten vor und vertritt klare Standpunkte, die in einem Maßnahmenkatalog zusammengefasst sind: Verbot der Werbung für Tabakprodukte, wirksame Primär- und Sekundärprävention, Erhöhung der Tabaksteuer, Verbot des Verkaufs von Zigaretten an Jugendliche unter 18 Jahren und Abschaffung der Tabakautomaten, Erhöhung der Gesundheitsversicherungsbeiträge für Raucher, finanzielle Unterstützung der Krankenkassen für die Raucherprophylaxe bei Kindern und Jugendlichen, Selbstverpflichtung der Ärzte zur aktiven Mitwirkung, dringender Appell an die Politiker, ihre Position zur Tabakindustrie zu überdenken und entsprechend Stellung zu beziehen, Anerkennung von Nikotin als Sucht erzeugende Substanz.

„Tabakentwöhnung“ ist ein lesenswertes, spannendes, informatives und nützliches Buch, Argumentationshilfe für jeden überzeugten Nichtraucher, ein Schatzkästlein zur Erstellung von Aufklärungs- und Schulungsmaterialien und eine dringende Empfehlung für jeden, der Prävention zur Vermeidung des Rauchens betreiben will und Rehabilitation bei Rauchern durchführen muss. 